\title{
Strain rate and temperature dependent strain localization of dynamically stretched lightweight bars: from metal to polymer
}

\author{
Longhui Zhang $^{1 *}$, David Townsend ${ }^{1}$, Nik Petrinic ${ }^{1}$, and Antonio Pellegrino ${ }^{1}$ \\ ${ }^{1}$ Department of Engineering Science, University of Oxford, Parks Road, Oxford, OX1 3PJ, U.K.
}

\begin{abstract}
This work studies the dynamic strain localization and constitutive relationship of a Ti3Al2.5V alloy in jet engine containment system and a transparent polycarbonate conceived for aircraft canopy application by Digital Image Correlation (DIC) technique from quasi-static condition to high strain rates at different temperatures. The responses of two materials show significant strain rate and temperature sensitivities. Observations of Ti3Al2.5V alloy show that the dynamic local strain rate can reach values up to $1000 \%$ of the nominal strain rate in the necking zone. However, dynamic local strain rate of polycarbonate soars up during strain softening then decreases rapidly with necking propagation, and eventually becomes $20 \%$ of the nominal strain rate until fracture. Appropriate viscoplastic constitutive models are determined for both materials, which are incorporated in finite element simulations to reveal the trend of dynamic local strain rate evolution in dynamic tensile tests. The present work shows two different kinds of strain localization in typical lightweight materials, which should be addressed carefully from Split Hopkinson Tension Bar (SHTB) tests.
\end{abstract}

\section{Introduction}

SHTB technique has been widely proposed for the characterization of the tensile response of materials at high strain rates since Harding et al. [1]. However, the analysis of SHTB test is subjected to non-uniform deformation such as the necking localization. Lightweight metals and polymers have been increasingly applied in aerospace engineering, which would be subjected to high-speed deformation in service. It's important to understand the high rate constitutive response and the strain localization in the materials. Quasi-static to high strain rate tensile tests, with the assistance of DIC technique to accurately measure the strain history, are carried out to investigate the dynamic strain localization in a Ti3 A12.5V alloy and a polycarbonate (PC). Likewise, the sensitivities of strain rate and temperature on the constitutive response and failure are also studied, in order to guide the design of aerospace components.

\section{Experimental protocol}

The $3 \mathrm{~mm}$ gauge diameter dog-bone shape specimens of Ti3A12.5V alloy with $8 \mathrm{~mm}$ gauge length and $3 \mathrm{~mm}$ gauge length, and the $4 \mathrm{~mm}$ gauge diameter dog-bone shape

*Corresponding author: longhui.zhang@eng.ox.ac.uk and longhui.z@alumni.technion.ac.i1 
polycarbonate specimens with $8 \mathrm{~mm}$ gauge length and $4 \mathrm{~mm}$ gauge length, were used to achieve various strain rates. A slightly bigger diameter of $4 \mathrm{~mm}$ in polycarbonate aims to increase the force signal measurement. The quasi-static and medium rate tensile tests were carried out on a screw-driven Zwick machine and Instron machine equipped with highspeed cameras. The engineering strain of the specimen was directly measured from the gauge section via DIC analysis using commercial software Lavision Davis. The true local strain is defined as $\varepsilon_{\text {true }}=2 \cdot \ln \left(\frac{r_{o}}{r}\right)$, where $r_{o}$ and $r$ are the original and current radius of the minimum cross section of the specimen gauge section. High strain rate tensile tests were conducted using a bespoke SHTB [2], synchronized by a high-speed Kirana camera. Tests of Ti3 Al2.5V alloy at $100{ }^{\circ} \mathrm{C}$ and $200{ }^{\circ} \mathrm{C}$ were performed using an induction heater, while the dynamic tests of polycarbonate at $75{ }^{\circ} \mathrm{C}$ and $110{ }^{\circ} \mathrm{C}$ were conducted by using an environmental chamber.



(a)

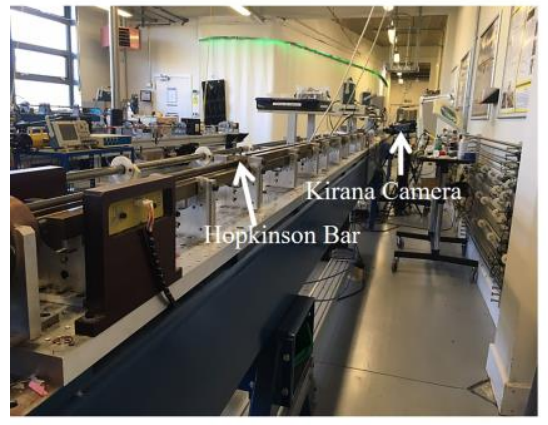

(b)



(c)

Fig.1 Experimental Setup (a) Schematic of the experimental setup (b) Image of SHTB System (b) Image of Kirana and the environmental chamber setups for elevated temperature tests

\section{Experimental results}

Fig. 2a and Fig.2b compare the engineering stress-strain relationships of Ti3Al2.5V alloy and polycarbonate under quasi-static condition 0.001-0.01/s, medium rates 9-150 /s, and high strain rates $1200-2700 / \mathrm{s}$. The ultimate engineering stress of Ti3Al2.5V alloy is 703 MPa under quasi-static condition, beyond which the engineering stress decreases due to necking localization with the failure engineering strain of 0.29 . The strain rate effect on the flow stress and failure strain is apparent for Ti3Al2.5V alloy. For polycarbonate, the engineering stress-strain experiences an initial peak stress about $63 \mathrm{MPa}$, followed by strain softening during necking, and the cold drawing process with apparent strain hardening. The failure engineering strain is about 1.24. The initial engineering peak stress increases from $63 \mathrm{MPa}$ under quasi-static loading to $73 \mathrm{MPa}$ at nominal strain rate of $9 / \mathrm{s}$ and $105 \mathrm{MPa}$ at 
nominal strain rate of 2700 /s. The strains to failure are not apparently strain rate dependent, compared to the flow stress which presents significant strain rate dependency.

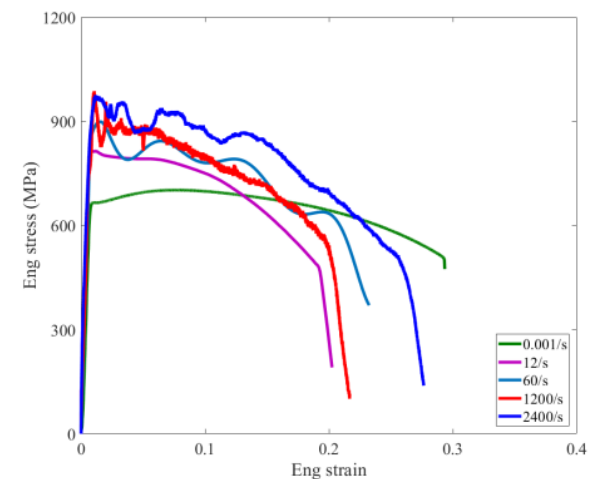

(a) $\mathrm{Ti} 3 \mathrm{~A} 12.5 \mathrm{~V}$

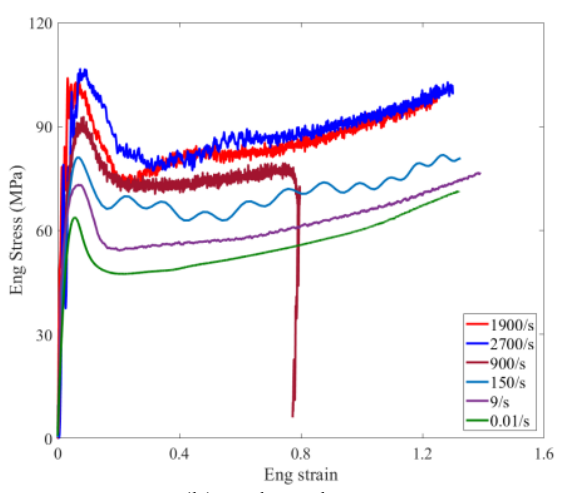

(b) Polycarbonate

Fig.2 Engineering stress-strain relationship of two materials from low to high strain rates

Fig. 3 compares the engineering stress-strain relationships of Ti3 Al2.5V and polycarbonate at different temperatures at high strain rates of 1200-2700/s. The flow stress decreases with increasing temperature, while the engineering strain to failure increases for two materials.

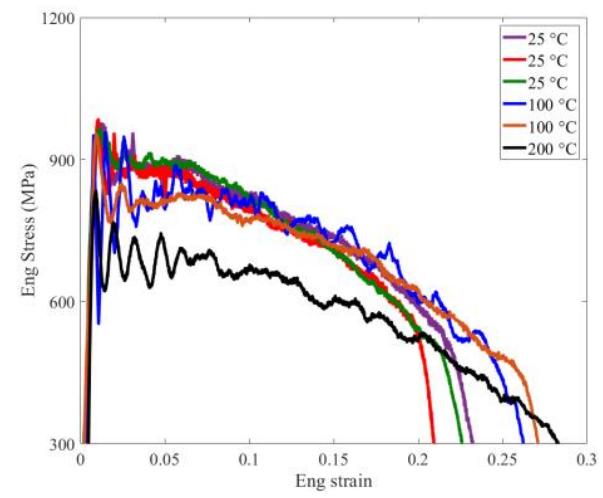

(a) $\mathrm{Ti} 3 \mathrm{Al} 2.5 \mathrm{~V}$



(b) Polycarbonate

Fig.3 Engineering stress-strain relationship at high strain rates and elevated temperatures

Fig.4a shows the local true strain rate in Ti3 Al2.5V alloy increases up to approximately 10000 /s [3-6]. However, for the polycarbonate, the dynamic local strain rate initially increases dramatically to almost $200 \%$ of the nominal strain rate during strain localization, followed by a rapid drop and finally tends to stay at strain rate of $400 / \mathrm{s}$ which is approximately $20 \%$ of the nominal strain rate until fracture. Therefore, the nominal strain rate firstly underestimates the local strain rate and then overestimates the local strain rate up to fracture. No matter for the test of the metallic rod or polymeric rod, the nominal strain rate from SHTB analysis fails to reflect the local strain rate and should be addressed carefully. 


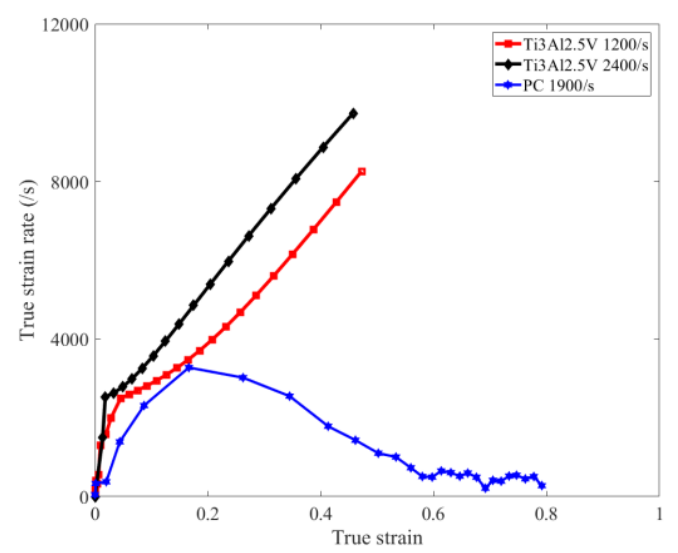

Fig.4 Local strain rate evolutions for two materials at high strain rates

Fig.5 shows the dynamic deformation process of two materials. The strain localization can be seen at 76 us for Ti3 Al2.5V specimen, which is followed by further localization until the fracture of the specimen at 182 us. For polycarbonate, one can observe the necking stage at 177 us, beyond which the localization propagates to the whole gage section and the uniform deformation continues to 677 us. Regarding the failure process of polycarbonate, a small crack is noticeable in the gauge section close to the output side (right side) of the specimen at 817 us. The crack propagates to the whole current cross-section, resulting in the final fracture of the gage section at 927 us. In addition to the two main separation parts, the fracture cross-section splits into several fragments.

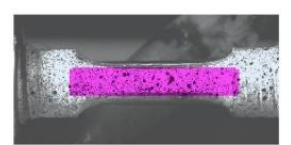

(0 us)

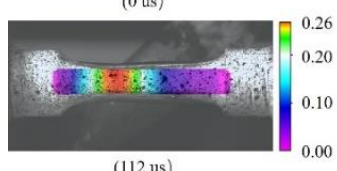

(112 us)

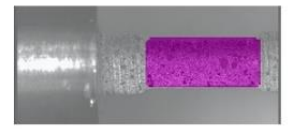

(0 us)

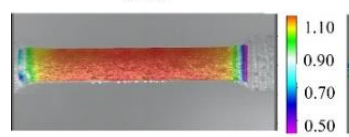

(677 us)

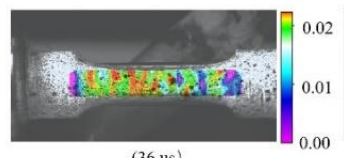

(36 us)



(146 us)

(a)

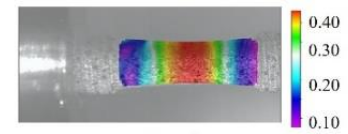

(177 us)

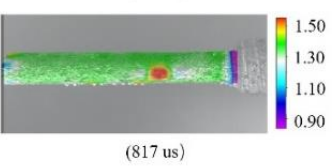

(b)
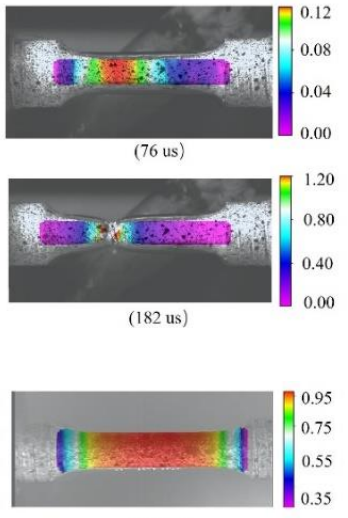

(477 us)

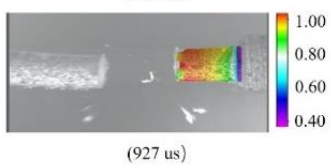

Fig.5 Dynamic deformation process with DIC at high strain rates of 1200-1900 /s. (a) Ti3Al2.5V (b) polycarbonate. Axial engineering strain filed is shown. 


\section{Numerical Simulations}

Appropriate constitutive models [5] are constructed based on the experimental results, which is also implemented in ABAQUS by VUMAT subroutine for the simulations of dynamic tensile tests of $8 \mathrm{~mm}$ length-3mm diameter gauge section specimens. The experimental force history can be predicted by the numerical macro force data for Ti3 Al2.5 alloy in Fig. 6a. The local strain rate is influenced by necking during dynamic tensile deformation and can reach values up to 10000 /s which is much higher than the nominal strain rate shown in Fig.6b.



(a)Typical macro force-time history of at $1200 / \mathrm{s}$

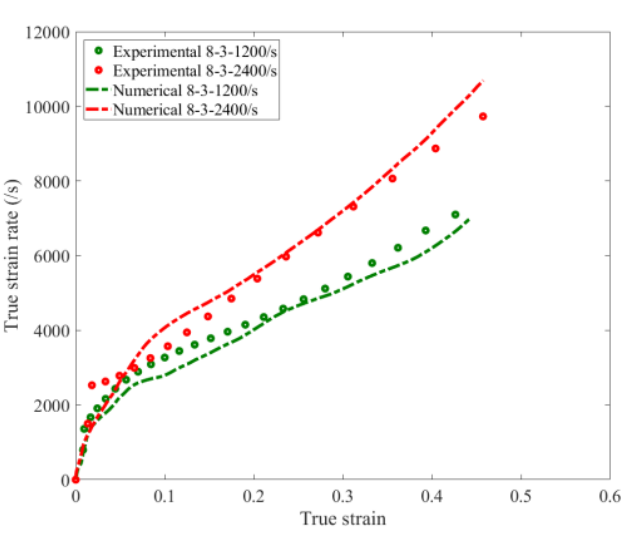

(b) Local strain rate evolutions

Fig. 6 Comparison of (a) macro force history and (b) local strain rate histories between the simulations and experiments at high strain rates at $25^{\circ} \mathrm{C}$ for Ti3 Al2.5V alloy

Fig.7a compares the experimental and numerical tensile force-displacement data at nominal strain rates of $1900 / \mathrm{s}$ for $8 \mathrm{~mm}$ gauge length polycarbonate specimen. The numerical global tensile force-displacement relationship agrees with the experimental measurements. A peak force is observed in the response indicating the following dynamic strain localization. Fig. $7 \mathrm{~b}$ shows the simulated strain rate histories to reveal the trend of experimentally observed local strain rate evolutions. The local strain rate immediately increases to about twice nominal strain rate due to strain localization. Then the local strain rate decreases rapidly as the localization propagates throughout the gauge section. Identical trends can be seen in both numerical and experimental local strain rate histories. Beyond 400 us, the local strain rates stay at a low strain rate region about $20 \%$ of the nominal strain rate, indicating the local strain rate is not constant during the dynamic tensile deformation of polycarbonate. 


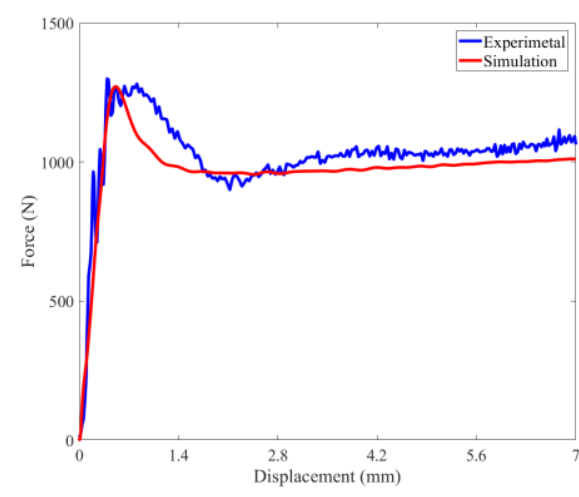

(a)

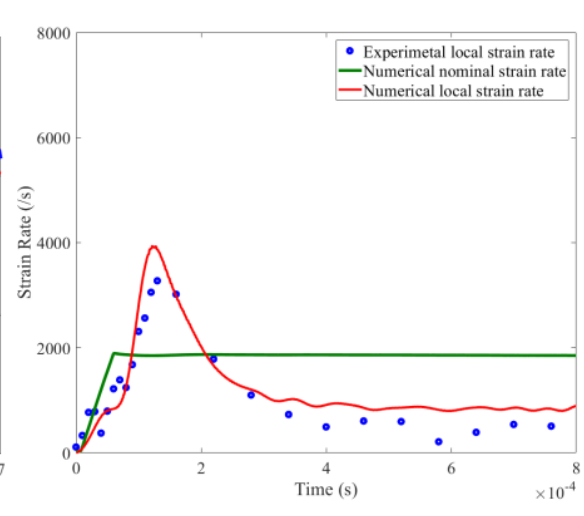

(b)

Fig.7 Comparison of (a) macro force-displacement and (b) local strain rate histories between the numerical results and experimental measurements for polycarbonate

\section{Conclusion}

The mechanical responses of Ti3Al2.5V alloy and polycarbonate are greatly influenced by the strain rate and temperature. The Ti3Al2.5V specimen shows high strain rates up to 10000 /s due to necking localization. The dynamic local strain rate of polycarbonate jumps during strain softening, then decreases with necking propagation, and eventually becomes constant at strain rate of approximately 400 /s until fracture occurs. The trends of dynamic local strain rate evolution of two materials are reproduced in numerical simulations. The present investigation clearly shows two different types of dynamic strain localization in two typical lightweight materials, which should be addressed carefully from SHTB tests.

\section{References}

[1] J. Harding, E. Wood, J. Campbell, Tensile testing of materials at impact rates of strain, J. Mech. Eng. Sci, 2, 88, (1960)

[2] R. Gerlach, C. Kettenbeil, N. Petrinic, A new split Hopkinson tensile bar design, Int. J. Impact Eng, 50, 63, (2012)

[3] G. Mirone, D. Corallo, R. Barbagallo, Experimental issues in tensile Hopkinson bar testing and a model of dynamic hardening, Int. J. Impact Eng, 103, 180, (2017)

[4] G. Mirone, R. Barbagallo, F. Giudice, locking of the strain rate effect in hopkinson bar testing of a mild steel, Int. J. Impact Eng, 130, 97, (2019)

[5] L. Zhang, A. Pellegrino, D. Townsend, N. Petrinic, Strain rate and temperature dependent strain localization of a near $\alpha$ titanium alloy, Int. J. Impact Eng, 145, 103676, (2020)

[6] L. Zhang, G. Gour, N. Petrinic, A. Pellegrino, Rate dependent behaviour and dynamic strain localisation of three novel impact resilient titanium alloys: Experiments and modelling, Mater. Sci. Eng. A, 771, 138552, (2020) 